\title{
Uterine Tachysystole with Prolonged Deceleration Following Nipple Stimulation for Labor Augmentation
}

\author{
Narasimhulu DM, Zhu L
}

Department of Obstetrics and Gynecology

Maimonides Medical Center

$4802,10^{\text {th }}$ ave, Brooklyn, New York.

\begin{abstract}
Breast stimulation for inducing uterine contractions has been reported in the medical literature since the $18^{\text {th }}$ century. The American college of Obstetricians and Gynecologists (ACOG) has described nipple stimulation as a natural and inexpensive nonmedical method for inducing labor.
\end{abstract}

Deepa Maheswari Narasimhulu

Department of Obstetrics and Gynecology

We report on a 37 year old P2 with a singleton pregnancy at 40 weeks gestation who developed tachysystole with a prolonged deceleration after nipple stimulation for augmentation of labor. Initial resuscitative measures, including oxygen by mask, a bolus of intravenous fluids and left lateral positioning, did not restore the fetal heart rate to normal. After the administration of Terbutaline $250 \mathrm{mcg}$ subcutaneously, the tachysystole resolved and the fetal heart rate recovered after five minutes of bradycardia.

Most trials of nipple stimulation for induction or augmentation of labor have had small study populations, and no conclusions could be drawn about the safety of nipple stimulation, though its use is widespread. While there have been a few reports of similar complications during nipple stimulation for contraction stress testing, there are no previous reports of tachysystole with sustained bradycardia following nipple stimulation for labor augmentation.

In this report, we draw attention to the dangers of nipple stimulation so that providers will be aware of this potential complication.

\section{KEY WORDS}

Deceleration, labor, nipple stimulation, uterine tachysystole.

\section{INTRODUCTION}

Breast stimulation for inducing uterine contractions has been reported in the medical literature since the $18^{\text {th }}$ century. ${ }^{1}$ The American college of Obstetricians and Gynecologists (ACOG) has described nipple stimulation as a natural and inexpensive nonmedical method for inducing labor. ${ }^{2}$ We report on a patient with an otherwise uncomplicated pregnancy with spontaneous rupture of membranes who experienced an episode of tachysystole with a prolonged deceleration that followed nipple stimulation. The episode did not resolve with initial resuscitative measures, but ultimately, after tocolytic therapy (subcutaneous terbutaline) there was resolution of the tachysystole and recovery of the fetal heart rate.

\section{CASE REPORTS}

A 37 year old P2 with a singleton pregnancy at 40 weeks gestation presented with complaints of contractions and leaking amniotic fluid. She had two previous vaginal deliveries and her current pregnancy was uncomplicated. She was found to be contracting every 5 minutes and the fetal status was reassuring (Fig 1). The pelvic exam revealed a cervix that was $3 \mathrm{~cm}$ dilated, $60 \%$ effaced and soft with leakage of clear amniotic fluid. The patient ambulated for few hours. When she returned, the pelvic exam was unchanged and her contractions were occurring every five minutes. She was then admitted to the labor and delivery unit for delivery. 


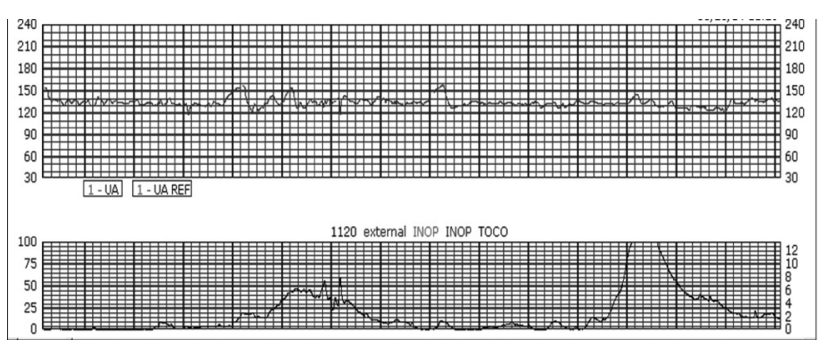

Figure 1. At initial presentation: Fetal heart rate was category I reactive with contractions every 5 minutes

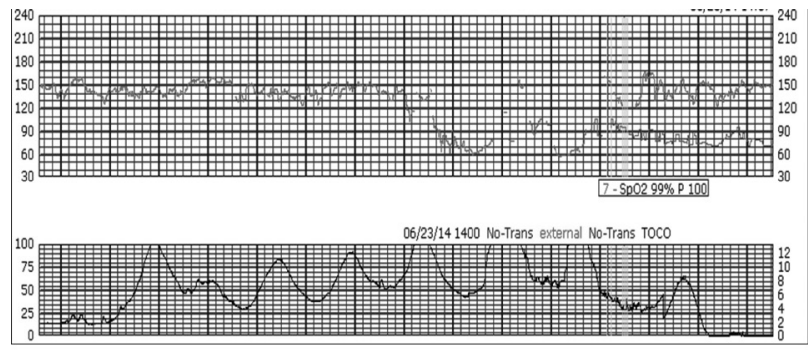

Figure 2. Shortly after admission: Nipple stimulation resulting in tachysystole and prolonged deceleration with a nadir of 60 beats per minute and lasting five minutes

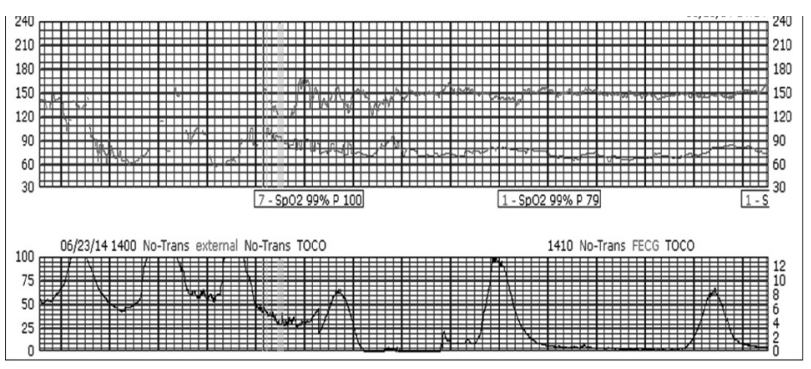

Figure 3. Resolution of tachysystole and recovery of the fetal heart rate after tocolytic therapy

Shortly thereafter, the patient developed tachysystole with contractions every minute and a prolonged deceleration with a nadir of 60 beats per minute (Fig 2). Resuscitative measures including oxygen by mask, a bolus of intravenous fluids and left lateral positioning failed. The pelvic exam at that time revealed that the cervix was $4 \mathrm{~cm}$ dilated and $80 \%$ effaced. Scalp stimulation did not elicit a response. Terbutaline $250 \mathrm{mcg}$ was administered subcutaneously following which the tachysystole resolved and the fetal heart rate recovered after a total of five minutes of bradycardia (Fig 3). Subsequently, the patient admitted to performing nipple stimulation because she thought her contractions were infrequent and not painful. When questioned as to how she knew about nipple stimulation, the patient stated that at the time of her previous birth in China, she had been instructed by her provider to perform nipple stimulation to improve the contractions. The patient was then counseled about the risks of nipple stimulation, and urged to not repeat the intervention. Following that episode, the fetal heart rate remained category I. Over the next several hours the contractions increased in frequency and the patient delivered a healthy male infant weighing 3820 grams with Apgar scores of $8 / 10$ at 1 minute and 9/10 at 5 minutes.

\section{DISCUSSION}

To the best of our knowledge (after searching Pubmed and Google scholar) we are reporting the first case of uterine tachysystole with sustained bradycardia following nipple stimulation for labor augmentation. Nipple stimulation causes release of oxytocin from the posterior pituitary, thereby increased uterine contractility. Stimulation of the nipple by manual rolling of the nipple as well as by the breast pump has been used for performing contraction stress tests, induction or augmentation of labor and for reducing blood loss in the third stage of labor. Since the amount of oxytocin released is not controlled, performing nipple stimulation may result in unpredictable uterine response with resultant complications including uterine tachysystole with or without fetal heart rate changes.

Most of the data on safety of nipple stimulation is from studies where it was performed for contraction stress testing, where the fetus is being monitored. This data cannot be extrapolated to the safety of nipple stimulation when performed to induce or augment labor as these contractions may be stronger resulting in uterine tachysystole and fetal bradycardia, especially when performed at home in an unmonitored setting.

The ACOG recommendation on nipple stimulation for induction of labor is based on a systematic review of six trials that included 719 women, and that compared breast stimulation with no intervention. They reported a significant decrease in the number of women not in labor at 72 hours, but only among women with a favorable bishop's score. None of the women had uterine tachysystole with or without fetal heart rate changes, and there were no differences in of rates of meconium stained amniotic fluid or cesarean delivery. ${ }^{2}$ All of those studies except the study by Damania et al. enrolled low risk women and all three perinatal deaths reported with nipple stimulation occurred in that study. ${ }^{3}$ The authors concluded that further research was needed to evaluate the safety of nipple stimulation. ${ }^{4}$

There have been two randomized trials comparing nipple stimulation and oxytocin for labor augmentation, neither of which found any difference in the length of labor between groups. Curtis et al. reported a similar incidence of adverse fetal and maternal events including decelerations, ${ }^{5}$ decreased beat-to-beat variability, meconium staining or uterine hypertonicity. However, the number of patients who had tachysystole with fetal heart rate changes was not reported. Stein et al. reported no adverse fetal effects in either study group. ${ }^{6}$

Most of these trials had small study populations such that no conclusions can be drawn about the safety of nipple stimulation for labor induction and augmentation, however its use is widespread. An informal survey of midwives and midwifery students practicing in various settings in and around New York City revealed that five of 15 sites surveyed regularly employed nipple stimulation for labor 
augmentation. Three of those sites were hospital labor and delivery units. ${ }^{7}$ While no provider would administer oxytocin for labor induction or augmentation to a pregnant woman at home, it is surprising that nipple stimulation is being performed at home.

\section{REFERENCES}

1. Marques KA, Amis JE, Devente JE. Uterine hyperstimulation by intravenous access in a mammary vein: a case report and literature review. Breastfeed Med. 2012;7(3):194-6.

2. ACOG Committee on Practice Bulletins - Obstetrics. ACOG Practice Bulletin No. 107: Induction of labor. Obstet Gynecol. 2009; 114:38697.

3. Damania KK, Natu U, Mhatre PN, Mataliya M, Mehta AC, Daftary SN. Evaluation of two methods employed for cervical ripening. J Postgrad Med. 1992;38(2):58-9.

4. Kavanagh J, Kelly AJ, Thomas J. Breast stimulation for cervical ripening and induction of labour. Cochrane Database Syst Rev. $2005 \mathrm{Jul}$ 20;(3):CD003392.
Although there are no previous reports of tachysystole with sustained bradycardia following nipple stimulation for augmenting labor, there have been a few reports of similar complications while performing nipple stimulation for contraction stress testing. ${ }^{8,9}$ We now report similar dangers from nipple stimulation in the context of augmentation of labor.

5. Curtis P, Resnick JC, Evens S, Thompson CJ. A comparison of breast stimulation and intravenous oxytocin for the augmentation of labor. Birth. 1999;26(2):115-22.

6. Stein JL, Bardeguez AD, Verma UL, Tegani N. Nipple stimulation for labor augmentation. J Reprod Med. 1990;35(7):710-4.

7. Razgaitis EJ, Lyvers AN. Management of protracted active labor with nipple stimulation: a viable tool for midwives? J Midwifery Womens Health. 2010;55(1):65-9.

8. Viegas OA, Arulkumaran S, Gibb DM, Ratnam SS. Nipple stimulation in late pregnancy causing uterine hyperstimulation and profound fetal bradycardia. Br J Obstet Gynaecol. 1984;91(4):364-6.

9. Schellpfeffer MA, Hoyle D, Johnson JW. Antepartal uterine hypercontractility secondary to nipple stimulation. Obstet Gynecol. $1985 ; 65(4): 588-91$. 